\title{
Vasohibin: the feedback on a new inhibitor of angiogenesis
}

\author{
Robert S. Kerbel \\ Sunnybrook and Women's College Health Sciences Centre and the University of Toronto, Toronto, Ontario, Canada.
}

\begin{abstract}
Angiogenesis is regulated in large part by the balance of various proangiogenic stimulators, such as VEGF, and a diverse group of endogenous inhibitors of angiogenesis, most of which are extrinsic to endothelial cells. With respect to the latter, until recently, none have appeared to be induced as a consequence of a specific, self-regulating, feedback inhibition response. A new inhibitor, called vasohibin, has been uncovered. Vasohibin is selectively induced in endothelial cells by proangiogenic stimulatory growth factors such as VEGF; it appears to operate as an intrinsic and highly specific feedback inhibitor of activated endothelial cells engaged in the process of angiogenesis (see the related article beginning on page 898).
\end{abstract}

\section{The yin and yang of angiogenesis}

The early years of angiogenesis research were dominated by intensive searches for the precise growth factors that stimulate this process of new blood vessel formation from preexisting mature and quiescent vasculature (1). The discovery of bFGF and especially VEGF in the mid- to late 1980s (2) were seminal events that significantly advanced the field $(1,2)$. But very soon afterward it became apparent that the angiogenic universe not only revolves around the action of such stimulators but also depends on a large number of diverse, endogenous protein inhibitors $(1,3-7)$. Some of these appear to be highly specific for endothelial cells, but are not, for the most part, synthesized by these cells, i.e., they are extrinsic inhibitors. Strong hints of the possible fundamental importance of such inhibitors for regulating angiogenesis were published in the mid- to late 1970s by Langer, Folkman, and colleagues who extracted a functional inhibitor from cartilage $(8,9)$, a tissue that is poorly vascularized. Since then, literally dozens of endogenous inhibitors have been described; some of these are listed in Table 1. The induction of angiogenesis in tumors (frequently referred to as the "angiogenic switch") is thought to be the consequence of a change in the local balance of stimulators and inhibitors; when the ratio is skewed in

Nonstandard abbreviations used: PEDF, pigment epithelium-derived factor; TSP-1, thrombospondin-1.

Conflict of interest: The author has declared that no conflict of interest exists.

Citation for this article: J. Clin. Invest. 114:884-886 (2004). doi:10.1172/JCI200423153. favor of the inhibitors, the switch is off, or at least in "low mode" if one thinks of a rheostat as an analogy, rather than an on-and-off switch. In contrast, the switch is turned on (or the reostat turned up) when the balance shifts toward the stimulators $(6,10)$. Many of these principles were discovered by cancer researchers, since sustained, pathologic angiogenesis is necessary for progressive expansion of tumor mass, as first hypothesized and shown by Folkman and colleagues $(11,12)$. A combination of genetic mutations, such as oncogene activation and inactivation of tumor suppressor genes, can induce and upregulate stimulators such as VEGF while concurrently downregulating inhibitors such as thrombospondin-1 (TSP-1) $(1,10)$. The same dual effects can be induced by microenvironmental-mediated factors, such as hypoxia $(1,2)$.

So far, none of the identified inhibitors of angiogenesis appears to operate on the basis of specific feedback inhibition mechanisms. Feedback inhibition is a classic, self-regulating type of control mechanism known to affect, for example, the production of various peptide hormones or the biosynthesis of amino acids. Thus, feedback inhibition of a single biosynthetic pathway can result when the designated end product suppresses the first enzyme in the pathway that is unique to the synthesis of the end product and therefore controls its own cellular level. Since physiologic angiogenesis is normally a finely tuned, tightly regulated process and endothelial cells are known to have extremely slow turnover times - except when called upon to form new blood vessels, after which they abruptly shut down - this suggests the existence of some kind of endothelial cell-specific feedback inhibitor control mechanism.

In this issue of the JCI, Watanabe et al. (13) report the details of a new regulator of angiogenesis, called vasohibin, which has some operational features of such an endothelial cell-specific feedback inhibitor. Unlike some other angiogenesis inhibitors, such as TSP-1, which may be secondarily induced by other known antiangiogenic drugs or various treatments such as frequently administered low-dose (metronomic) chemotherapy (14-17), or doxycycline (18), vasohibin is induced over time in vascular endothelial cells by angiogenesis stimulators, especially VEGF. The purified protein, which is not glycosylated, appears capable of inhibiting angiogenesis in vivo when tested using a variety of different assays. Similarly, it inhibits several endothelial cell functions in vitro that are relevant to neovascularization. Antisense oligonucleotides specific for vasohibin shift a VEGF-induced, bell-shaped, dose response in a manner suggestive of blocking a feedback inhibitory response. For example, the effect of relatively high levels of VEGF, which can actually suppress endothelial cell migration in vitro (in contrast to lower levels which are growth stimulatory), was reversed by such antisense treatment. When the gene encoding vasohibin (KIAA1036) was transfected into tumor cells, their growth was blocked in vivo but not in vitro, consistent with a hypothetical role in regulating angiogenesis. Experiments with a very limited number of different cell types suggest vasohibin is produced by and acts exclusively on endothelial cells. Time will tell whether this specificity will hold.

\section{Feedback inhibition of angiogenesis: a precedent?}

The results reported by Watanabe et al. (13) are exciting, but the notion of angiogenesis stimulators setting in motion a chain of events within endothelial cells that lead to the cells' eventual growth inhibition or even death is perhaps not entirely without precedent. Volpert et al. reported that the apoptosis-signaling Fas/CD95 receptor 
Table 1

Some endogenous inhibitors of angiogenesis

Inhibitor
TSP-1 and -2
IFN- $\alpha$ and $-\beta$
Angiostatin
Endostatin
Tumstatin
Canstatin
16 kDa fragment of prolactin
2-Methoxyestradial
PEDF
VEGI

VEGI, vascular endothelial growth inhibitor.

is induced by stimulators of angiogenesis and, because of this, can potentially specifically sensitize the stimulated endothelial cells to FasL-mediated apoptosis, which can occur through the action of angiogenesis inhibitors such as TSP-1 or pigment epithelium-derived factor (PEDF) since both can induce FasL in endothelial cells (19). So the Volpert study appears to be a precedent showing that cooperation of proangiogenic and angiogenesis inhibition factors causes eventual inhibition of angiogenesis. But in the case of Volpert et al., the inhibitors appear to be extrinsic to endothelial cells.

\section{Looking ahead: questions and more questions}

Vasohibin's discovery and unique properties raise many questions that are not only

\section{Figure 1}

Control of angiogenesis by the action of extrinsic angiogenesis inhibitors (antiangiogenic factors), an intrinsic endothelial cell inhibitor (vasohibin), and extrinsic stimulators of angiogenesis such as VEGF and bFGF. Many of the extrinsic type inhibitors, some of which are listed in Table 1, may act to keep established mature blood vessels in a quiescent state (left side of the diagram); they may also contribute to pathologic, sprouting angiogenesis as a result of their downregulation and/or suppression, thus allowing stimulators such as VEGF or bFGF to act more efficiently. The intrinsic inhibitor, vasohibin, is induced in endothelial cells at later stages of sprouting vessel formation and acts in some fashion as a feedback mechanism to limit excessive angiogenesis (right side of the diagram), e.g., by directly interacting with endothelial cells in sprouting vessels or perhaps by direct interaction with and neutralization of the stimulator(s) which induced vasohibin in endothelial cells (13). pertinent to the regulation of angiogenesis but also to therapeutic angiogenesis and antiangiogenic therapy. How does vasohibin actually work to block angiogenesis (see Figure 1)? Specifically, does it interfere with the function or expression of VEGFR-2 itself (the main signaling receptor for VEGF-mediated angiogenesis) or downstream signaling events in endothelial cells induced by VEGF after it binds to VEGFR-2? Might it even directly bind to VEGF in the circulation? Should any of these possibilities be implicated by future studies, the evidence for a true, specific, feedback inhibition of angiogenesis, in this case induced by VEGF, would be strengthened. Is it possible that the therapeutic use of growth factors such as bFGF and VEGF to promote angiogenesis for the treatment of ischemic conditions would be compromised by induction of vasohibin? On the other side of the coin, is it the case that agents such as anti-VEGF antibodies mediate at least some of their antiangiogenic effects by causing a downregulation of vasohibin in endothelial cells? Testing such drugs in vasohibin knockout mice, if and when available, should provide an answer. With respect to regulation of sustained tumor angiogenesis, vasohibin expression should be downregulated, pre-

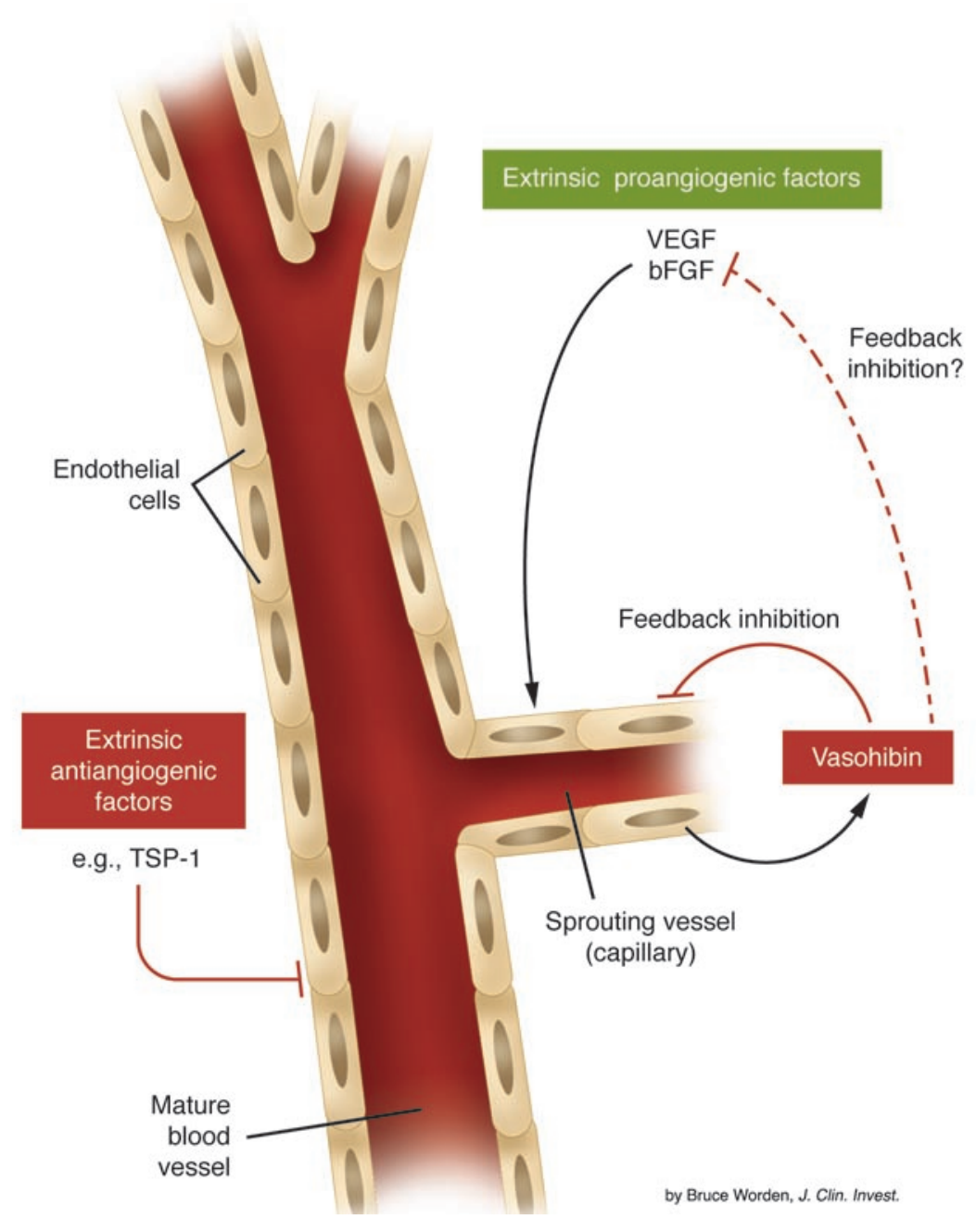


sumably, despite high local concentrations of stimulators in the tumor microenvironment, such as VEGF. How might this happen? Watanabe et al. (13) provide a potential answer on the basis of an experiment in which hypoxia - which is commonly associated with solid tumors - was found to suppress the VEGF-mediated induction of vasohibin in cultured endothelial cells. What about the effects on vasohibin by other endothelial cell-reactive inhibitors such as TSP-1? Might they actually suppress its expression, possibly leading to a form of induced or acquired resistance to various endogenous angiogenesis inhibitors (20-22)? Finally, if vasohibin acts on mature vascular endothelial cells, what are its effects on putative circulating peripheral blood endothelial progenitor cells (23)? In this regard, Watanabe et al. report that despite the lack of a classic secretion signal sequence, a cleaved form of vasohibin is apparently released from endothelial cells. This means it may freely circulate and, provided that sufficient concentrations can be attained in the blood, it could potentially affect either circulating endothelial cells or their progenitor subset cells, assuming such cells actually express a vasohibin receptor or binding element.

Clearly, these questions are only the tip of the vasohibin iceberg. As answers begin to unfold we shall learn whether or not an exciting new field in endothelial cell biology and angiogenesis has just been launched.
Address correspondence to: Robert S. Kerbel, Sunnybrook and Women's College Health Sciences Centre and the University of Toronto, S-217 Research Building, 2075 Bayview Avenue, Toronto, Ontario M4N 3M5, Canada. Phone: (416) 480-5711; Fax: (416) 4805884; E-mail: robert.kerbel@swchsc.on.ca.

1. Kerbel, R.S., and Folkman, J. 2002. Clinical translation of angiogenesis inhibitors. Nat. Rev. Cancer. 2:727-739.

2. Ferrara, N., Hillan, K.J., Gerber, H.P., and Novotny, W. 2004. Discovery and development of bevacizumab, an anti-VEGF antibody for treating cancer. Nat. Rev. Drug Discov. 3:391-400.

3. Dameron, K.M., Volpert, O.V., Tainsky, M.A., and Bouck, N. 1994. Control of angiogenesis in fibroblasts by p53 regulation of thrombospondin-1. Science. 265:1582-1584.

4. Kalluri, R. 2002. Discovery of type IV collagen noncollagenous domains as novel integrin ligands and endogenous inhibitors of angiogenesis. Cold Spring Harb. Symp. Quant. Biol. 67:255-266.

5. Lindner, D.J. 2002. Interferons as antiangiogenic agents. Curr. Oncol. Rep. 4:510-514.

6. Hanahan, D., and Folkman, J. 1996. Patterns and emerging mechanisms of the angiogenic switch during tumorigenesis. Cell. 86:353-364.

7. Sage, H. 1999. Pieces of eight: bioactive fragments of extracellular proteins as regulators of angiogenesis. Trends Cell Biol. 7:182-186.

8. Langer, R., Brem, H., Falterman, K., Klein, M., and Folkman, J. 1976. Isolations of a cartilage factor that inhibits tumor neovascularization. Science. 193:70-72.

9. Langer, R., Conn, H., Vacanti, J., Haudenschild, C., and Folkman, J. 1980. Control of tumor growth in animals by infusion of an angiogenesis inhibitor. Proc. Natl. Acad. Sci. U. S. A. 77:4331-4335.

10. Bouck, N., Stellmach, V., and Hsu, S.C. 1996. How tumors become angiogenic. Adv. Cancer Res. 69:135-174.

11. Folkman, J. 1985. Tumor angiogenesis. Adv. Cancer Res. 43:175-203.
12. Folkman, J. 1971. Tumor angiogenesis: therapeutic implications. N. Engl. J. Med. 285:1182-1186.

13. Watanabe, K., et al. 2004. Vasohibin as an endothelium-derived negative feedback regulator of angiogenesis. J. Clin. Invest. 114:898-907. doi:10.1172/ JCI200421152.

14. Hanahan, D., Bergers, G., and Bergsland, E. 2000. Less is more, regularly: metronomic dosing of cytotoxic drugs can target tumor angiogenesis in mice. J. Clin. Invest. 105:1045-1047.

15. Kerbel, R.S., and Kamen, B.A. 2004. Antiangiogenic basis of low-dose metronomic chemotherapy. Nat. Rev. Cancer. 4:423-436.

16. Bocci, G., Francia, G., Man, S., Lawler, J., and Kerbel, R.S. 2003. Thrombospondin-1, a mediator of the antiangiogenic effects of low-dose metronomic chemotherapy. Proc. Natl. Acad. Sci. U. S. A. 100:12917-12922.

17. Hamano, Y., et al. 2004. Thrombospondin-1 associated with tumor microenvironment contributes to low-dose cyclophosphamide-mediated endothelial cell apoptosis and tumor growth suppression. Cancer Res. 64:1570-1574.

18. Kalas, W., et al. 2003. Restoration of thrombospondin 1 expression in tumor cells harbouring mutant ras oncogene by treatment with low doses of doxycycline. Biochem. Biophys. Res. Commun. 310:109-114.

19. Volpert, O.V., et al. 2002. Inducer-stimulated Fas targets activated endothelium for destruction by anti-angiogenic thrombospondin-1 and pigment epithelium-derived factor. Nat. Med. 8:349-357.

20. Yu, J.L., Rak, J.W., Coomber, B.L., Hicklin, D.J., and Kerbel, R.S. 2002. Effect of p53 status on tumor response to antiangiogenic therapy. Science. 295:1526-1528.

21. Filleur, S., et al. 2001. In vivo mechanisms by which tumors producing thrombospondin 1 bypass its inhibitory effects. Genes Dev. 15:1373-1382.

22. Filleur, S., et al. 2003. SiRNA-mediated inhibition of vascular endothelial growth factor severely limits tumor resistance to antiangiogenic thrombospondin- 1 and slows tumor vascularization and growth. Cancer Res. 63:3919-3922.

23. Rafii, S., Lyden, D., Benezra, R., Hattori, K., and Heissig, B. 2002. Vascular and haematopoietic stem cells: novel targets for anti-angiogenesis therapy? Nat. Rev. Cancer. 2:826-835.

\title{
IRS2 takes center stage in the development of type 2 diabetes
}

\author{
Matthew J. Brady
}

Department of Medicine, Committee on Molecular Metabolism and Nutrition, University of Chicago, Chicago, Illinois, USA.

\begin{abstract}
The etiology of type 2 diabetes is characterized by obesity, insulin and leptin resistance, and compensatory $\beta$ cell hyperplasia followed by islet degeneration, resulting in the eventual dysregulation of glucose and lipid homeostasis. The recent identification of insulin receptor substrate 2 (IRS2) as a central player in the pathophysiology of many of these processes suggests a potentially unifying molecular link underlying the initiation and progression of type 2 diabetes (see the related articles beginning on pages 908 and 917).
\end{abstract}

Nonstandard abbreviations used: IRS, insulin receptor substrate.

Conflict of interest: The author has declared that no conflict of interest exists.

Citation for this article: J. Clin. Invest. 114:886-888 (2004). doi:10.1172/JCI200423108.
Diabetes mellitus is characterized by an inability of the body to efficiently utilize circulating nutrients. Normally, in response to elevation of plasma glucose, insulin is secreted by $\beta$ cells in the islets of Langerhans and promotes energy uptake, metabolism, and storage. Type 2 diabetes is preceded by the development of insulin resistance in target tissues (1), but the molecular causes of its initiation and progression remain unclear. Obesity is the predominant risk factor for the onset of insulin resistance, although other genetic and environmental factors also contribute (2). Islets initially compensate for the loss of insulin sensitivity by expanding $\beta$ cell mass and insulin secretory capacity, but over time, they cannot continue to meet the chronic stress of increased insulin demand. The loss of $\beta$ cells and reduction in circulating insulin levels occur 